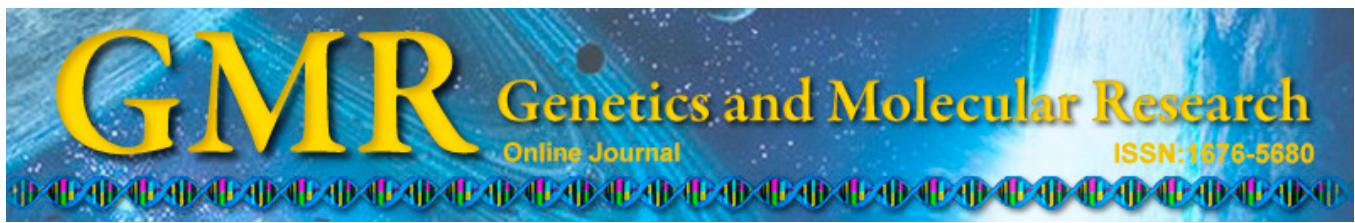

\title{
Clinical analysis of patients with myeloperoxidase antineutrophil cytoplasmic antibody-associated vasculitis
}

\author{
Z.W. Shuai, Y.F. Lv, M.M. Zhang and Z.Y. Hu \\ Department of Rheumatology, \\ First Affiliated Hospital of Medical University of Anhui, Hefei, China \\ Corresponding author: Z.W. Shuai \\ E-mail: shuaizongwen_zw@163.com
}

Genet. Mol. Res. 14 (2): 5296-5303 (2015)

Received October 1, 2014

Accepted December 9, 2014

Published May 18, 2015

DOI http://dx.doi.org/10.4238/2015.May.18.22

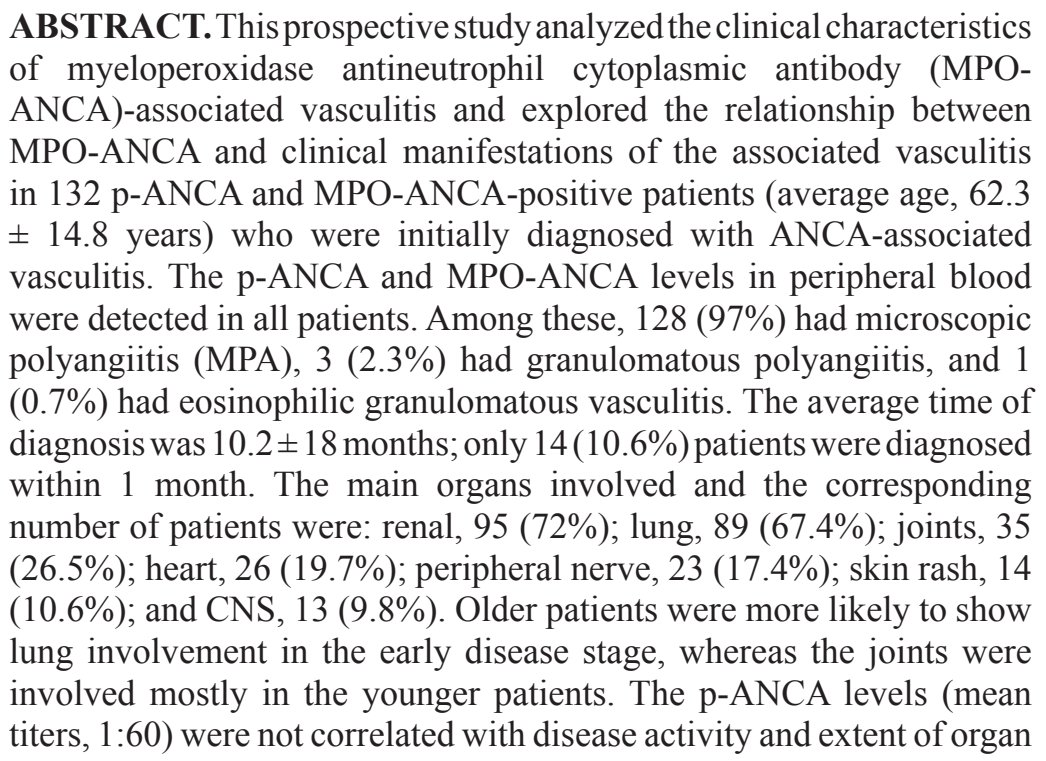


involvement, and the MPO-ANCA levels were positively correlated with disease activity, but had no correlation with the extent of organ involvement. MPO-ANCA vasculitis is a common occurrence in China; it mainly involves the elderly and presents as clinical manifestations of MPA. However, the multiple organ damage is not specific leading to delay in diagnosis. MPO-ANCA may play a pathogenic role in the associated vasculitis, and the diverse clinical manifestations might be related with the different characteristics of MPO-ANCA.

Key words: Antineutrophil cytoplasmic antibody; Clinical damage; Antimyeloperoxidase antibody; ANCA-associated vasculitis;

Pathogenic mechanism

\section{INTRODUCTION}

Antineutrophil cytoplasmic antibody (ANCA)-associated vasculitis (AAV), which encompasses granulomatous polyangiitis (GPA), microscopic polyangiitis (MPA), and eosinophilic granulomatosis with polyangiitis (EGPA) (Jennette et al., 2013), is a fatal systemic disease. Myeloperoxidase (MPO) is one of the main self-antigens for ANCA. The association of peri-nuclear ANCA (p-ANCA) with MPA and EGPA has been detected by indirect immunofluorescence (IIF). According to previous studies, the incidence of GPA in the European population is $>60 \%$ (Reinhold-Keller et al., 2005), that of MPA in China and Japan is $79-83 \%$ (Chen et al., 2005; Fujimoto et al., 2011), and that of MPO-ANCA is as high as 59-94\% (Oh et al., 2009; Ahn et al., 2012). Inducing AAV in an immunized animal model with MPO-ANCA or MPO antigen in a previous study (Xiao et al., 2002; Little et al., 2005; Roth et al., 2013) suggested that MPO-ANCA may play a pathogenic role in AAV. Therefore, MPO-ANCA may be the main antibody associated with AAV in Chinese patients. MPO-AAV is not rare in China (Chen et al., 2005). However, it has received little attention, and the lack of awareness has led to delayed clinical diagnosis and treatment of this disease. Here, $132 \mathrm{MPO}-\mathrm{AAV}$ patients were studied prospectively to further improve the understanding of this disease, and to discuss the possible role of MPO-ANCA in clinical damage associated with AAV.

\section{MATERIAL AND METHODS}

\section{Research subjects}

From July 2008 to July 2013, a total of 132 patients (age, $62.3 \pm 14.8$; range, 4-85 years) from the First Affiliated Hospital of Medical University of Anhui were included in the study. The male:female ratio was 43:89. IIF was used to detect p-ANCA $(+)$ in all patients, and ELISA was used to detect MPO-ANCA $(+)$; the PR3-ANCA $(+)$ or ANA $(+)$ patients were excluded. All patients met the American College of Rheumatology GPA and EGPA classification criteria and the definition of MPA, GPA, or EGPA, according to the 2012 Chapel Hill Consensus Conference (CHCC) (Leavitt et al., 1990; Masi et al., 1990; Jennette et al., 2013). All 132 patients were newly diagnosed with AAV and were in the active disease period, except for those in whom the diagnosis of connective tissue diseases including rheumatoid arthritis, 
systemic lupus erythematosus, and systemic sclerosis was confirmed. Clinical data were collected in all patients, and the Birmingham vasculitis activity score (BVAS) was evaluated. Peripheral blood was collected and cryopreserved at $-80^{\circ} \mathrm{C}$.

\section{ANCA detection method}

\section{p-ANCA detection}

The human neutrophils were fixed with alcohol as the substrate (Oumeng Medical Laboratory Diagnosis Kit for Screening of Limited by Share Ltd., Germany); peripheral blood of p-ANCA $(+)$ patients was screened using the IIF method, and the antibody titer was measured.

\section{MPO-ANCA detection}

According to the experimental steps of the kit, preliminary screening of MPO-ANCA $(+)$ in p-ANCA $(+)$ patients was performed with immunobinding dot assay (Oumeng Medical Laboratory Diagnosis Limited by Share Ltd. Kit). Quantitative detection of MPO-ANCA was performed using ELISA. We added $50 \mu \mathrm{L}$ human MPO antigen in each hole of the 96-hole enzyme plate (Sigma-Aldrich, USA; diluting the concentration of $10 \mu \mathrm{g} / \mathrm{mL}$ ) and placed it at $4^{\circ} \mathrm{C}$ overnight; the remaining steps were the same as with conventional ELISA. Each plate had the known MPO-ANCA (+) serum as the positive normal control, a negative control, and a blank control. Using the od value $\mathrm{x}+2 s$ of normal negative controls as the upper limit, we excluded negative patients based on ELISA and analyzed the relationship between ANCA and the involved organs and BVAS.

\section{Statistical analysis}

Clinical and laboratory data of all patients were analyzed with the SPSS 19.0 software (IBM, USA). $\mathrm{P}<0.05$ was considered to be statistically significant.

\section{RESULTS}

\section{Patient characteristics}

All 132 participants were newly diagnosed as AAV. They were admitted in 8 different departments of our hospital based upon their clinical presentation as follows: 43 patients $(32.6 \%)$ in renal internal medicine, $40(30.3 \%)$ in rheumatology and immunology, $33(25 \%)$ in the respiratory department of internal medicine, 5 each (3.8\%) in the departments of infection and digestion of internal medicine, $3(2.3 \%)$ in endocrinology, $2(1.5 \%)$ in pediatrics, and 1 $(0.8 \%)$ in ear-nose-throat. Among these, 128 patients had MPA (97\%), 3 had GPA (2.3\%), and 1 had EGPA (0.7\%). The average age of the patients was $62.3 \pm 14.8$ (4-85) years and elderly patients $>60$ years of age accounted for $63.6 \%$ (84 patients). The time period from onset of AAV-related symptoms to diagnosis was $10.2 \pm 18(0.3-120)$ months. Only $10.6 \%$ of the patients (14) were diagnosed by ANCA examination within 1 month after disease onset; $51.5 \%$ (68) were diagnosed within 3 months; and 72.0\% (95) were diagnosed within 1 year. All patients were in the active disease stage, and the average BVAS was $17.7 \pm 6.5(6-35)$ points. 


\section{Clinical manifestations}

Sixty-seven (50.8\%) patients had fever; 38 (28.8\%) of these had temperature higher than $38.5^{\circ} \mathrm{C}$. A total of $129(97.7 \%)$ patients had fatigue symptoms, and the body weight $(83.3 \%)$ of 110 patients had decreased by a varying extent. The multi-organ involvement is shown in Table 1.

Table 1. Multiple organ damage in 132 patients with MPO-AAV [N (\%)].

\begin{tabular}{cccccccccccccccc}
\hline Kidney & Lungs & Joint & Heart & $\begin{array}{c}\text { Peripheral } \\
\text { nerve }\end{array}$ & Skin & $\begin{array}{c}\text { Central } \\
\text { nervous }\end{array}$ & Muscle & $\begin{array}{c}\text { Digestive } \\
\text { tract }\end{array}$ & Ear & Eye & Nose & Throat & Oral Liver Total \\
\hline 95 & 89 & 35 & 26 & 23 & 14 & 13 & 9 & 8 & 6 & 5 & 4 & 3 & 2 & 1 & 132 \\
$(72.0)$ & $(67.4)$ & $(26.5)$ & $(19.7)$ & $(17.4)$ & $(10.6)$ & $(9.8)$ & $(6.8)$ & $(6.1)$ & $(4.5)$ & $(3.8)$ & $(3.0)$ & $(2.3)$ & $(1.5)$ & $(0.7)$ & $(100)$ \\
\hline
\end{tabular}

Among the multiple organs involved, renal damage was the most common (95/132 patients, $72 \%$ ) and hematuria was the most common symptom (79/95 patients, $83.2 \%$ ) followed by proteinuria (68/95 patients, $71.6 \%$ ); 42 patients had acute renal failure, and renal biopsy of 23 patients was consistent with the renal lesion associated with MPA. Ten (12.4\%) of 89 patients with pulmonary damage had hemoptysis; CT revealed inflammatory exudate in 74 patients $(77.9 \%)$, followed by pleural effusion/pleurisy in 34 patients $(38.2 \%)$ and lung nodules/fibrosis in 19 patients (21.3\%). Biopsy examination in 2 patients revealed pulmonary inflammatory lesions in one and necrotic granulomatous disease in the other. Joint damage was mainly manifested as peripheral arthritis (red and swollen joints and activity limitations). Twenty-six patients experienced cardiac damage, 12 (46.15\%) had pericarditis/pericardial effusion, 9 (34.6\%) had chronic heart failure/cardiomyopathy, 1 had aortic insufficiency, and 1 had new onset arrhythmia. Peripheral neuropathy was confirmed by electromyography, and the most common nerve lesions were single $(9 / 23,39.1 \%)$, followed by peripheral neuritis $(6 / 29,20.7 \%)$. Thirteen patients had central nervous system damage, with MRI examination revealing stroke as the most common diagnosis (12 patients); this included 2 patients with symptoms of epilepsy. Electromyography or muscle biopsy confirmed that 9 patients had muscle lesions, and 3 had obvious symptoms of myasthenia gravis. The most common manifestation for digestive tract damage is abdominal pain; 4 patients had vomiting/diarrhea. Recent decline in hearing was found to be due to otitis media and nerve involvement. A comparison of the involved major organ (the kidney, lung, heart, and joint, as shown in Table 1) and the disease course and patient age is presented in Table 2.

\begin{tabular}{|c|c|c|c|c|c|c|c|c|}
\hline & \multicolumn{2}{|c|}{ Kidney } & \multicolumn{2}{|c|}{ Lungs } & \multicolumn{2}{|c|}{ Joint } & \multicolumn{2}{|c|}{ Heart } \\
\hline & Yes & No & Yes & No & Yes & No & Yes & No \\
\hline $\mathrm{N}$ & 95 & 37 & 89 & 43 & 35 & 97 & 26 & 106 \\
\hline \multicolumn{9}{|l|}{ Age } \\
\hline Mean (years) & $63.7 \pm 13.6$ & $58.7 \pm 17.4$ & $65.8 \pm 11.2$ & $55.1 \pm 18.6$ & $57.3 \pm 17.8$ & $64.1 \pm 13.3$ & $65.7 \pm 11.0$ & $61.4 \pm 15.6$ \\
\hline $\begin{array}{l}\text { T value } \\
\mathrm{P} \text { value } 0.082\end{array}$ & -1.755 & $0.001 *$ & -3.478 & $0.021 *$ & 2.335 & 0.192 & -1.312 & \\
\hline \multicolumn{9}{|l|}{ Course } \\
\hline Mean (months) & $10.6 \pm 19.2$ & $9.5 \pm 14.8$ & $6.2 \pm 9.6$ & $18.5 \pm 26.8$ & $9.8 \pm 23.3$ & $10.4 \pm 15.8$ & $13.5 \pm 25.8$ & $9.4 \pm 18.6$ \\
\hline $\mathrm{T}$ value & -0.308 & & 2.920 & & 0.184 & & -1.041 & \\
\hline $\mathrm{P}$ value & 0.795 & & $0.005 *$ & & 0.854 & & 0.300 & \\
\hline
\end{tabular}




\section{Laboratory data}

The total white blood cell count in the peripheral blood was $10.5 \pm 6.2 \times 10^{9} / \mathrm{L}$, and the neutrophil percentage was $84.0 \pm 68.5 \%$. The total white cell count in 72 patients $(54.6 \%)$ and the neutrophil granulocyte ratio in 73 patients $(55.3 \%)$ was higher than the normal range. The hemoglobin concentration of the patients was $92.3 \pm 21.9 \mathrm{~g} / \mathrm{L} ; 84.1 \%$ of the patients (111) had anemia of varying severity, $80.3 \%$ (106) had normal-cell normochromic anemia, and the hemoglobin concentration of $50.8 \%$ of the patients $(67)$ was less than $90 \mathrm{~g} / \mathrm{L}$. The platelet count was $260.1 \pm 119.6 \times 10^{9} / \mathrm{L}$, of which $29(22 \%)$ were in a higher than normal range. The erythrocyte sedimentation rate (ESR) and C-reactive protein (CRP) were $79.7 \pm 35.3 \mathrm{~mm} / \mathrm{h}$ and $66.4 \pm 70.9 \mathrm{mg} / \mathrm{L}$, respectively. The ESR of $96.2 \%$ of the patients (127) and CRP of $84.1 \%$ of the patients (111) were higher than normal.

\section{Correlation between MPO-ANCA and clinical damage}

The IIF detected patients' peripheral blood p-ANCA titers in the range of 1:20 to $1: 160$, with the average titer as 1:60. We performed a correlation analysis of peripheral blood p-ANCA and MPO-ANCA levels with BVAS and the involved organs, respectively. The results are shown in Table 3.

\begin{tabular}{|c|c|c|c|c|c|}
\hline & \multirow[t]{2}{*}{$\mathrm{N}$} & \multicolumn{2}{|c|}{ p-ANCA } & \multicolumn{2}{|c|}{ MPO-ANCA } \\
\hline & & $\mathrm{r}$ & $\mathrm{P}$ & $\mathrm{r}$ & $\mathrm{P}$ \\
\hline BVAS & 132 & 0.013 & 0.884 & 0.258 & $0.003^{*}$ \\
\hline Involvement extent $\boldsymbol{\Lambda}^{\boldsymbol{\Delta}}$ & 132 & 0.087 & 0.152 & 0.022 & 0.802 \\
\hline
\end{tabular}

Spearman rank correlation analysis; ${ }^{*} \mathrm{P}<0.05 ;{ }^{\star}$ the extent of multiple organ involvement as seen in Table 1.

\section{DISCUSSION}

Several previous studies have shown that although MPO-ANCA and PR3-ANCA were associated with AAV, they combined the disparate antigens in neutrophil cytoplasm, and their associated AAV showed different clinical manifestations and genetic backgrounds. According to Millet et al. (2013) MPO-AAV and PR3-AAV may be two independent diseases and should be studied separately. Existing evidence supports MPO-ANCA, rather than PR3-ANCA, as the pathogenic antibody of AAV (Xiao et al., 2002; Little et al., 2005; Schlieben et al., 2005; Roth et al., 2013). In China, MPO-ANCA-associated MPA is the main type of AAV. To investigate the characteristics of clinical damage associated with MPO-AAV and its relationship with MPO-ANCA, we only enrolled those who were MPO-ANCA $(+)$ in this study. Detection of p-ANCA with IIF and detection of MPO-ANCA with ELISA can more accurately diagnose MPO-AAV patients than detection of p-ANCA or MPO-ANCA alone (Perel et al., 2013).

This study enrolled 132 patients with MPO-AAV from the hospital, with 2.2 patients on average enrolled monthly, over 5 years. All patients were selected from one hospital and did not include any clinic and MPO-ANCA (-) patients. The actual number of patients with this disease may be higher as MPO-AAV is not rare in China. Because of the complex diver- 
sity of the initial clinical manifestations, patients were admitted in 6 different clinical departments besides the departments of nephropathy and rheumatism. Increased awareness of this condition among multiple disciplines is therefore essential.

The onset age for MPO-AAV is within the range of 4-85 years; however, it occurs mainly in the elderly patients (mean age, 62 years). The clinical manifestations of this disease are widespread and involve various organs, mainly the kidneys, lungs, and joints (Table 1). This is consistent with the main clinical departments (Renal Internal Medicine, Internal Medicine, and Respiratory Department of Rheumatology); the patients were admitted based on the primary diagnosis. The onset age, gender, and major organ damage distribution of patients were consistent with previous reports (Chen et al., 2005; Oh et al., 2009; Ahn et al., 2012). The clinical manifestations (including decreased fatigue, fever, and decrease in weight) of multi-system damage in MPO-AAV and routine laboratory abnormalities, which included increased total peripheral blood cell and neutrophil counts, anemia, and elevated ESR and CRP, all lack specificity. Especially in the elderly, symptoms such as hearing loss, numbness of the limbs, and cerebral infarction were often neglected, which may be the main reason for the average diagnosis time of 10 months. One and 3 months after the onset, the diagnosed patient rates were only 10.6 and $51.5 \%$, respectively. While the clinical manifestations in some of the MPA patients were acute fatal pulmonary hemorrhage and/or irreversible nephritis seen early in the disease course and progressing rapidly (in this study, there were 42 patients with acute renal failure and 10 with pulmonary hemorrhage), the effective treatment time is short. Therefore, improving awareness and vigilance regarding this disease is important for early diagnosis and treatment.

Further analysis of the relationship between the clinical damage (kidney, lungs, joints, and heart) and patients' age and course of disease revealed that age and disease course do not have a significant effect on the kidney and heart involvement. Older age or prolonged illness did not increase the clinical damage to the kidney and heart. The age of patients with pulmonary damage was significantly higher than that of patients without lung damage, and the course of disease was shorter, which suggested that elderly patients experienced early lung damage, or more serious lung damage (such as pulmonary hemorrhage) leading to early death. In fact, 7 patients died within 6 months in this group, of which 5 died of pulmonary hemorrhage or severe pulmonary infiltration with severe infection. The age of those with joint damage was lower than that of patients without joint damage; this suggested that more joint involvement occurred in the non-elderly patients; however, joint involvement was not related to the length of disease course.

Among the MPO-AAV patients, MPA accounted for $97 \%$, with 3 patients diagnosed with GPA [PR3-ANCA (-)]; MPO-ANCA may also be present in some of the patients with GPA (Kallenberg, 2011). Previous studies have reported that there was a stronger correlation between PR3-ANCA and the disease activity of AAV (BVAS), while the relationship between MPO-ANCA and BVAS was not consistent with the previous report. Han et al. (2003) reported that the titer of MPO-ANCA can be used as a predictor of recurrence and aggravation of AAV. In a previous study, Mahler et al. (2012) demonstrated that BVAS is not related to the MPO-ANCA levels, and its relationship with PR3-ANCA depends on the detection methods. The analysis results of this study (Table 3) showed that p-ANCA was independent of organ involvement and multi-system vasculitis activity scope in MPO-AAV patients. The other autoimmune antibodies in p-ANCA may recognize the non-MPO antigens of neutrophils such as elastase, cathepsin G, lysozyme, and bactericidal/permeability increasing protein) (Radice et al., 2013); these antibodies may affect the relation study of p-ANCA titers and pathogenicity. 
Table 3 demonstrates the positive correlation of MPO-ANCA levels with BVAS (the severity of clinical damage), and suggests that MPO-ANCA may be the pathogenic antibody of MPOAAV. Table 3 also shows that the MPO-ANCA levels were not correlated with the extent of organ involvement. Previous studies have reported that according to the differences in MPO antigen epitope specificity of ANCA, it may be associated with associated damage of different tissues and organs (Suzuki et al., 2007; Roth et al., 2013). In this study, no correlation was found between the level of MPO-ANCA and extent of organ involvement, which supports the fact that the diversity of clinical damage may be related to the differences in the epitope specificity of MPO-ANCA.

Roth et al. (2013) reported that the differences in the epitope specificity of MPOANCA determine its pathogenicity. In fact, MPO-ANCA can exist in a healthy human body with no pathogenicity, referred to as the non-pathogenic MPO-ANCA. On the other hand, ANCA (-) patients may suffer from AAV [i.e., the ANCA (-) AAV according to the 2012 $\mathrm{CHCC}$. There may be unmeasured MPO-ANCA in the serum of these patients (Cui et al., 2010; Jennette et al., 2013). One of the purposes of this study was to analyze the possible role of MPO-ANCA in the pathogenesis of MPO-AAV. We did not select the MPO-ANCA (-) cases and selected only the hospitalized patients with AAV disease activity. We also did not select the MPO-ANCA $(+)$ healthy individuals. Therefore, our conclusions need to be validated and improvised by further research. In addition, further investigation is needed to further explore the specific pathogenicity of different epitopes of MPO-ANCA, which may provide the clinical implications of diagnosis and treatment of patients with MPO-AAV.

\section{ACKNOWLEDGMENTS}

Research supported by the Chinese Medical Association Clinical Medical Special Fund Project (\#08010290107).

\section{REFERENCES}

Ahn JK, Hwang JW, Lee J, Jeon CH, et al. (2012). Clinical features and outcome of microscopic polyangiitis under a new consensus algorithm of ANCA-associated vasculitides in Korea. Rheumatol. Int. 32: 2979-2986.

Chen M, Yu F, Zhang Y and Zhao MH (2005). Clinical [corrected] and pathological characteristics of Chinese patients with antineutrophil cytoplasmic autoantibody associated systemic vasculitides: a study of 426 patients from a single centre. Postgrad. Med. J. 81: 723-727.

Cui Z, Zhao MH, Segelmark M and Hellmark T (2010). Natural autoantibodies to myeloperoxidase, proteinase 3, and the glomerular basement membrane are present in normal individuals. Kidney Int. 78: 590-597.

Fujimoto S, Watts RA, Kobayashi S, Suzuki K, et al. (2011). Comparison of the epidemiology of anti-neutrophil cytoplasmic antibody-associated vasculitis between Japan and the U.K. Rheumatology 50: 1916-1920.

Han WK, Choi HK, Roth RM, McCluskey RT, et al. (2003). Serial ANCA titers: useful tool for prevention of relapses in ANCA-associated vasculitis. Kidney Int. 63: 1079-1085.

Jennette JC, Falk RJ, Bacon PA, Basu N, et al. (2013). 2012 Revised international Chapel Hill consensus conference nomenclature of vasculitides. Arthritis Rheum. 65:1-11.

Kallenberg CG (2011). Pathogenesis of ANCA-associated vasculitides. Ann Rheum. Dis. 70 (Suppl 1): i59-i63.

Leavitt RY, Fauci AS, Bloch DA, Michel BA, et al. (1990). The American College of Rheumatology 1990 criteria for the classification of Wegener's granulomatosis. Arthritis Rheum. 33: 1101-1107.

Little MA, Smyth CL, Yadav R, Ambrose L, et al. (2005). Antineutrophil cytoplasm antibodies directed against myeloperoxidase augment leukocyte-microvascular interactions in vivo. Blood 106: 2050-2058.

Mahler M, Radice A, Yang W, Bentow C, et al. (2012). Development and performance evaluation of novel chemiluminescence assays for detection of anti-PR3 and anti-MPO antibodies. Clin. Chim. Acta 413: 719-726. 
Masi AT, Hunder GG, Lie JT, Michel BA, et al. (1990). The American College of Rheumatology 1990 criteria for the classification of Churg-Strauss syndrome (allergic granulomatosis and angiitis). Arthritis Rheum. 33: 1094-1100.

Millet A, Pederzoli-Ribeil M, Guillevin L, Witko-Sarasat V, et al. (2013). Antineutrophil cytoplasmic antibody-associated vasculitides: is it time to split up the group. Ann. Rheum. Dis. 72: 1273-1279.

Oh JS, Lee CK, Kim YG, Nah SS, et al. (2009). Clinical features and outcomes of microscopic polyangiitis in Korea. J. Korean Med. Sci. 24: 269-274.

Perel SB, Prain KM, Wilson RJ, Hogan PG, et al. (2013). Diagnostic value of distinguishing and reporting different perinuclear ANCA (P-ANCA) immunofluorescence patterns: A prospective study. Am. J. Clin. Pathol. 140: 184-192.

Radice A, Bianchi L and Sinico RA (2013). Anti-neutrophil cytoplasmic autoantibodies: methodological aspects and clinical significance in systemic vasculitis. Autoimmun. Rev. 12: 487-495.

Reinhold-Keller E, Herlyn K, Wagner-Bastmeyer R and Gross WL (2005). Stable incidence of primary systemic vasculitides over five years: results from the German vasculitis register. Arthritis Rheum. 53: 93-99.

Roth AJ, Ooi JD, Hess JJ, van Timmeren MM, et al. (2013). Epitope specificity determines pathogenicity and detectability in ANCA-associated vasculitis. J. Clin. Invest. 123: 1773-1783.

Schlieben DJ, Korbet SM, Kimura RE, Schwartz MM, et al. (2005). Pulmonary-renal syndrome in a newborn with placental transmission of ANCAs. Am. J. Kidney Dis. 45: 758-761.

Suzuki K, Kobayashi S, Yamazaki K, Gondo M, et al. (2007). Analysis of risk epitopes of anti-neutrophil antibody MPOANCA in vasculitis in Japanese population. Microbiol. Immunol. 51: 1215-1220.

Xiao H, Heeringa P, Hu P, Liu Z, et al. (2002). Antineutrophil cytoplasmic autoantibodies specific for myeloperoxidase cause glomerulonephritis and vasculitis in mice. J. Clin. Invest. 110: 955-963. 